\title{
Gecko-Inspired Adhesive Tape
}

A team of researchers from the USA and Germany has succeeded in developing a re-usable adhesive tape inspired by the feet of a gecko.

G eckos' feet are superior to adhesive tapes in one respect: even after repeated contact with dirt and dust, they perfectly adhere to smooth surfaces. Researchers at KIT and Carnegie Mellon University in Pittsburgh have now developed the first adhesive tape that not only adheres to a surface as reliably as the toes of a gecko, but also possesses similar self-cleaning properties. Using such a tape, food packaging or bandages, for example, might be opened and closed several times.

\section{Self-Cleaning Effect}

When moving forwards, a gecko's toes drag across part of the surface. As a result of this lateral friction contact, larger dirt particles are removed. Smaller particles deposit among the setae on the sole and in the skin folds below. In an experiment, the researchers have proved that both mechanisms provide for $95 \%$ of the self-cleaning effect. For their experiments, the scientists used elastic microhairs of variable sizes. Instead of dirt particles, they employed glass spheres of micrometre size, which they distributed on a smooth plate. To simulate the steps

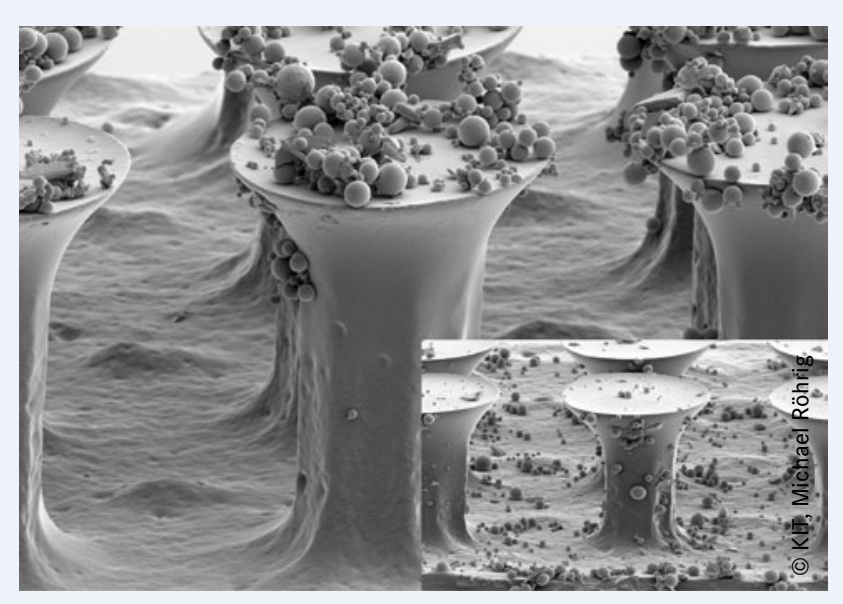

The Geckoinspired microhairs were photographed using a scanning electron microscope after cleaning by frictional contact with a smooth surface. made by a gecko, they pressed an artificial adhesive tape covered by microhairs onto the plate, shifted it laterally, and lifted the tape off again. This "loaddrag-unload" cycle was repeated several times and the adhesive force was measured each time.

\section{Glass Spheres to Simulate Dirt}

When the diameter of the spheres exceeded that of the microhairs, the adhesive force disappeared after the first contact ("load") - as is the case with an
This SEM image shows glass spheres between microhairs, which have a mushroom shape to increase adhesion.

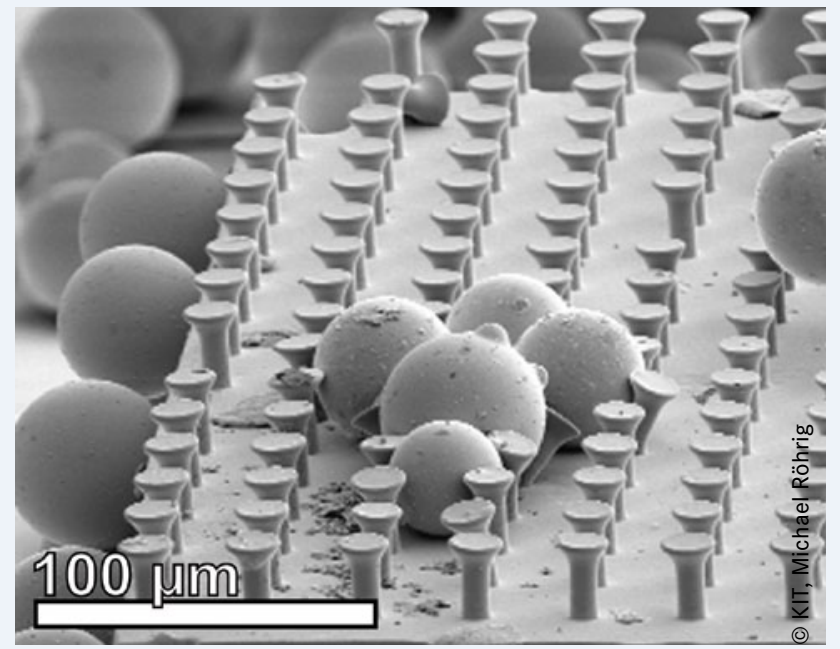

ordinary adhesive tape. After eight to ten test cycles, however, the gecko-inspired adhesive tape achieved 80 to 100 percent of its original power again. The researchers believe that this might be developed to become a low-cost alternative to hook and loop fasteners. Possible applications may be in the sports sector, medicine, the automotive industry or aerospace technology.

When the size of the spheres was smaller than the diameter of the microhairs, the researchers succeeded in restoring one third of the original adhesive force only. The perfect geckoinspired adhesive tape therefore requires fibres in the nanometre range, which are smaller than most dirt particles. The skin folds of the gecko have already been reproduced by wide grooves between narrow rows of hair. They provide enough space for the fine dust to deposit. Tests using real dirt particles of variable shape and size and particles made of various materials are to be carried out in the near future.

The scientists have published their findings in "Interface", the scientific journal of the Royal Society (DOI: rsif.2013.1205). 